\title{
MULTI-TUPLE SHILOV BOUNDARIES OF ALGEBRA TENSOR PRODUCTS
}

\author{
TOMA V. TONEV \\ (Communicated by Paul S. Muhly)
}

\begin{abstract}
We give a short proof of Basener Slodkowski theorem for multituple Shilov boundaries of tensor products of two uniform algebraas.
\end{abstract}

\section{INTRODUCTION}

Since their discovery in 1975 by Basener and Sibony the multi-tuple Shilov boundaries of commutative Banach algebras proved to be an essential tool in the investigating multi-dimensional analytic structures in algebra spectra. Results concerning the relationship of these boundaries and analyticity in algebra spectra have appeared often during the last fifteen years (e.g. Basener [1], Kramm [3], Kumagai [4], Sibony [5], Tonev [10,11], etc.). Various properties of multidimensional Shilov boundaries were investigated by Basener [1], Sibony [5], Slodkowski $[6,7]$, Tonev $[9,10]$ and others.

In 1983 Slodkowski [6] proved a conjecture originally due to Basener about expression of multi-tuple Shilov boundaries of a tensor product of two algebras in terms of multi-tuple Shilov boundaries of generating algebras. Namely:

Basener Slodkowski Theorem [7]. $\partial^{(n)} A \otimes B=\bigcup_{k=1}^{n} \partial^{(k)} A \times \partial^{(n-k-1)} B$.

This theorem generalizes the well known fact about the Shilov boundary of a tensor product of two algebras and also the corresponding result about multituple Shilov boundaries of polydisc algebras. Applications of this conjecture were pointed out by Kumagai [4]. Basener has proved the theorem for certain special cases only. Slodkowski's proof utilizes the theory of $q$-plurisubharmonic functions and a specific approximation procedure of algebra elements. It turns out that the notion of multi-tuple Shilov boundaries can be extended for function spaces as well. The first part of Slodkowski's proof in fact proves Basener's conjecture for the space $P(X)$, the closure of polynomials in $\mathbf{C}^{n}$ on a compact set $X \in \mathbf{C}^{n}$.

Received by the editors April 27, 1990 and, in revised form, April 7, 1991.

1980 Mathematics Subject Classification (1985 Revision). Primary 46J15.

Key words and phrases. Uniform algebra, algebra spectrum, Shilov boundary, multi-tuple Shilov boundaries, algebra tensor products. 


\section{Preliminaries}

Let $A$ be a uniform algebra over $\mathbf{C}$ with unit. That is $A$ is a separating closed subalgebra of the space of all continuous complex valued functions on some compact Hausdorff space $X$ which contains the constants and the norm of $f \in A$ is the maximum of $|f(x)|$ on $X$. As usual $\operatorname{sp} A$ denotes the maximal ideal space of $A$ and $\hat{f}$ denotes the Gelfand extension of a given function of $A$. The Shilov boundary $\partial A$ of $A$ is the smallest closed subset of $\operatorname{sp} A$ on which the Gelfand extensions of all functions of $A$ assume the maximums of their absolute values.

Denote by $V\left(f_{1}, \ldots, f_{n}\right)$ the vanishing set of a fixed $n$-tuple $\left(\widehat{f}_{1}, \ldots, \widehat{f}_{n}\right)$ over $\hat{A}$, i.e.

$$
V\left(f_{1}, \ldots, f_{n}\right)=\left\{m \in \operatorname{sp} A: \widehat{f}_{1}(m)=\widehat{f}_{2}(m)=\cdots=\widehat{f}_{n}(m)=0\right\} .
$$

By $A_{E}$ we denote the closure in $C(E)$ of restrictions of all elements of $\hat{A}$ on a fixed closed subset $E$ of $\operatorname{sp} A$.

Definition 1 (Basener, Sibony). The $n$-tuple Shilov boundary $\partial^{(n)} A$ of a commutative Banach algebra $A$ is the following subset of $\operatorname{sp} A$ :

$$
\partial^{(n)} A=\left[\bigcup\left\{\partial A_{V\left(f_{1}, \ldots, f_{n-1}\right)}:\left(f_{1}, \ldots, f_{n-1}\right) \in A^{n-1}\right\}\right]
$$

where [N] stands for the closure of $N$ in $\operatorname{sp} A, A^{0}=\{0\}$, and $A^{n}$ is the set of all $n$-tuples of elements of $A$ for $n \geq 1$ (see [1, 5], also [12]).

It is easy to check that

$$
\partial A=\partial^{(1)} A \subset \partial^{(2)} A \subset \partial^{(3)} A \subset \cdots \subset \partial^{(n)} A \subset \cdots \subset \operatorname{sp} A .
$$

We recall some of the basic properties of multi-tuple Shilov boundaries. An $n$-tuple $F=\left(\widehat{f}_{1}, \ldots, \widehat{f}_{n}\right) \in \widehat{A}^{n}$ is called regular if the functions $\widehat{f}_{1}, \ldots, \widehat{f}_{n}$ have no common zeros on $\operatorname{sp} A$, i.e. if $V\left(f_{1}, \ldots, f_{n}\right)=\varnothing$ or, in other words, if $0 \notin\left(\widehat{f}_{1}, \ldots, \widehat{f}_{n}\right)(\mathbf{s p} A)$.

Theorem 1 [9, Theorem 1]. $\partial^{(n)} A$ is the smallest closed subset of $\operatorname{sp} A$ on which every regular $n$-tuple $F=\left(\widehat{f}_{1}, \ldots, \widehat{f}_{n}\right) \in \widehat{A}$ assumes the minimum of its norm

$$
\|F(m)\|=\left(\sum_{j=1}^{n}\left\|\widehat{f}_{j}(m)\right\|^{2}\right)^{1 / 2}, \quad m \in \operatorname{sp} A .
$$

Given a subset $K$ of $\mathbf{C}^{n}, b K$ will denote in the sequel the topological boundary of $K$ with respect to the ambient $\mathbf{C}^{n}$.

Theorem 2 [9, Theorem 3]. $\partial^{(n)} A$ is the smallest closed subset of $\operatorname{sp} A$ such that the inclusion

$$
F\left(\partial^{(n)} A\right) \supset b F(\operatorname{sp} A)
$$

holds for every $n$-tuple $F=\left(\widehat{f}_{1}, \ldots, \widehat{f}_{n}\right) \in \widehat{A}^{n}$. 


\section{BASENER-SLODKOWSKI THEOREM}

Let $A$ and $B$ be two uniform algebras and let $A \otimes B$ denote the tensor product of $A$ and $B$. In other words $A \otimes B$ is isometrically isomorphic to the smallest closed subalgebra of $C(\operatorname{sp} A \times \operatorname{sp} B)$ which contains all monomials $f(x) g(y)$, where $f \in \widehat{A}, g \in \widehat{B}, x \in \operatorname{sp} A, y \in \operatorname{sp} B$. It is not hard to see that $\operatorname{sp} A \otimes B$ is homeomorphic to the set $\operatorname{sp} A \times \operatorname{sp} B$ and that

$$
\partial A \otimes B=\partial A \times \partial B
$$

The theorem under consideration may be stated as follows.

Theorem 3 (Basener-Slodkovski). $\partial^{(n)} A \otimes B=\bigcup_{k=1}^{n} \partial^{(k)} A \times \partial^{(n-k+1)} B$.

Proof. For the sake of simplicity we shall assume that $A=\widehat{A}$ and $B=\widehat{B}$. If we denote $\operatorname{sp} A$ by $X$ and $\operatorname{sp} B$ by $Y$, then $\operatorname{sp} A \otimes B$ is homeomorphic to $X \times Y$. The inclusion

$$
\partial^{(n)} A \otimes B \supset \bigcup \partial^{(k)} A \times \partial^{(n-k+1)} B
$$

is the easier one and can be checked without any trouble. The opposite inclusion

$$
\partial^{(n)} A \otimes B \subset \bigcup \partial^{(k)} A \times \partial^{(n-k+1)} B
$$

we shall prove in two steps. Denote for a while the set $\bigcup \partial^{(k)} A \times \partial^{(n-k+1)} B$ by $E$.

Step 1. Any function of type

$$
F(x, y)=\left(f_{1}(x), \ldots, f_{k}(x), g_{1}(y), \ldots, g_{n-k}(y)\right),
$$

where $f_{i}(x) \in A$ and $g_{j}(y) \in B, i=1, \ldots, k, j=1, \ldots, n-k$, satisfies the following inclusion

$$
b F(X \times Y) \subset F(E) .
$$

Indeed, let $\left(x_{0}, y_{0}\right) \in X \times Y$ and let $\|F(x, y)\| \neq 0$. Since

$$
\begin{aligned}
\|F(x, y)\|^{2} & =\left\|\left(f_{1}(x), \ldots, f_{k}(x), g_{1}(y), \ldots, g_{n-k}(y)\right)\right\|^{2} \\
& =\left\|\left(f_{1}(x), \ldots, f_{k}(x)\right)\right\|^{2}+\left\|\left(g_{1}(y), \ldots, g_{n-k}(y)\right)\right\|^{2}
\end{aligned}
$$

we have that

$$
\begin{aligned}
\left\|F\left(x_{0}, y_{0}\right)\right\|^{2} & \geq \min _{X \times Y}\|F(x, y)\|^{2} \\
& =\min _{X}\left\|\left(f_{1}(x), \ldots, f_{k}(x)\right)\right\|^{2}+\min _{Y}\left\|\left(g_{1}(y), \ldots, g_{n-k}(y)\right)\right\|^{2} .
\end{aligned}
$$

If $\left\|\left(g_{1}(y), \ldots, g_{n-k}(y)\right)\right\|$ vanishes on $Y$ then certainly the $k$-tuple $\left(f_{1}(x)\right.$, $\left.\ldots, f_{k}(x)\right)$ does not vanish on $X$ and

$$
\min _{X}\left\|\left(f_{1}(x), \ldots, f_{k}(x)\right)\right\|=\min _{\partial^{(k)} A}\left\|\left(f_{1}(x), \ldots, f_{k}(x)\right)\right\|
$$


by Theorem 1 , so that

$$
\begin{aligned}
\left\|F\left(x_{0}, y_{0}\right)\right\| & \geq \min _{\partial^{(k)} A \times V\left(g_{1}, \ldots, g_{n-k}\right)}\|F(x, y)\| \\
& =\min _{\partial^{(k)} A \times \partial B_{V\left(g_{1}, \ldots, g_{n-k}\right)}}\|F(x, y)\| \geq \min _{\partial^{(k)} A \times \partial^{(n-k+1)} B}\|F(x, y)\| .
\end{aligned}
$$

If $\left\|\left(f_{1}(x), \ldots, f_{k}(x)\right)\right\|$ vanishes on $X$, then certainly $\left(g_{1}(y), \ldots g_{n-k}(y)\right)$ does not vanish on $Y$ and we can obtain in a similar way that $\left\|F\left(x_{0}, y_{0}\right)\right\| \geq$ $\min _{\partial^{(k+1)} A \times \partial^{(n-k)} B}\|F(x, y)\|$. If both $\left(f_{1}(x), \ldots, f_{k}(x)\right)$ and $\left(g_{1}(y), \ldots\right.$, $\left.g_{n-k}(y)\right)$ do not vanish, then again as above we get that

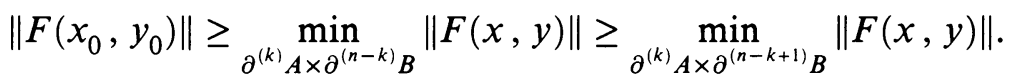

Consequently

$$
\left\|F\left(x_{0}, y_{0}\right)\right\| \geq \min _{\cup_{k=1}^{n} \partial^{(k)} A \times \partial^{(n-k+1)} B}\|F(x, y)\|=\min _{E}\|F(x, y)\|
$$

for any regular $n$-tuple $F$ of type (6). If we assume that (7) is violated for some $F$ of type (6) we can find a point $\left(x_{0}, y_{0}\right) \in X \times Y$ such that $F\left(x_{0}, y_{0}\right) \in$ $b F(X \times Y) \backslash F(E)$. Consequently we can find a point $Z_{0}$ in $\mathbf{C}^{n} \backslash \sigma(F)$ close enough to $F\left(x_{0}, y_{0}\right)$, such that the function $H=F-Z_{0}$ does not vanish on $X \times Y$ and at the same time does not satisfy the inequality (8) in contradiction with the result just proved. We conclude that the inclusion (7) is fulfilled for any $n$-tuple $F$ of type (6).

Step 2. The inclusion (7) holds for every mapping $F(x, y) \in(A \otimes B)^{2}$.

Proof. We prove the case $n=2$ of the statement. Let $F \in A^{k}, g \in B^{l}$ and let $P_{1}$ and $P_{2}$ be polynomials in $k$ and $l$ variables respectively. It is not difficult to check that

$$
\partial^{(j)} P(F(X)) \subset F\left(\partial^{(j)} P(X)\right)
$$

for each $j$ and for every $F \in A^{k}$. Therefore

$$
\begin{aligned}
b\left(P_{1}\right. & \left.(F, G), P_{2}(F, G)\right)(X \times Y)=b\left(P_{1}, P_{2}\right)(F(X) \times G(Y)) \\
& \subset\left(P_{1}, P_{2}\right)\left(\partial^{(2)} P(F(X) \times G(Y))\right) \\
& =\left(P_{1}, P_{2}\right)\left\{\left(\partial P(F(X)) \times \partial^{(2)} P(G(Y))\right) \cup\left(\partial^{(2)} P(F(X)) \times \partial P(G(Y))\right)\right\} \\
& \subset\left(P_{1}, P_{2}\right)\left\{\left(F(\partial A) \times G\left(\partial^{(2)} B\right)\right) \cup\left(F\left(\partial^{(2)} A\right) \times G(\partial B)\right)\right\} \\
& =\left(P_{1}, P_{2}\right)\left\{(F, G)\left(\partial A \times \partial^{(2)} B\right) \cup(F, G)\left(\partial^{(2)} A \times \partial B\right)\right\} \\
& =\left(P_{1}, P_{2}\right)(F, G)\left(\partial A \times \partial^{(2)} B \cup \partial^{(2)} A \times \partial B\right) \\
& =\left(P_{1}, P_{2}\right)(F, G)(E)=\left(P_{1}(F, G), P_{2}(F, G)\right)(E) .
\end{aligned}
$$

Since the mappings of type $\left(P_{1}(F, G), P_{2}(F, G)\right)$ are uniformly dense in $(A \otimes B)^{2}$ we conclude that $(7)$ holds for every function in $(A \otimes B)^{2}$. This proves the case $n=2$ of Theorem 3 because by Theorem $2, \partial^{(2)} A \otimes B$ is the 
smallest closed subset of $X \times Y$ for which (7) holds for any mapping from $(A \otimes B)^{2}$.

The proof of the general case $(n>2)$ of Theorem 3 follows the same lines as the proof of the case $n=2$.

\section{REFERENCES}

1. R. Basener, A generalized Shilov boundary and analytic structure, Proc. Amer. Math. Soc. 47 (1975), 98-104.

2. _ Boundaries for product algebras, preprint, 1975.

3. B. Kramm, Nuclearity (resp. schwarzity) helps to embed holomorphic structure into spectra (a survey), Banach Algebras and Several Complex Variables, Contemp. Math., vol. 32, Amer. Math. Soc., Providence, RI, 1983, pp. 143-162.

4. D. Kumagai, Subharmonic functions and uniform algebras, Proc. Amer. Math. Soc. 78 (1980), 23-29.

5. N. Sibony, Multi-dimensional analytic structure in the spectrum of a uniform algebra, Lecture Notes in Math., vol. 512, Springer-Verlag, New York, 1976, pp. 139-175.

6. Z. Slodkowski, Analytic multifunctions, q-plurisubharmonic functions and uniform algebras, Banach Algebras and Several Complex Variables, Contemp. Math., vol. 32, Amer. Math. Soc., Providence, RI, 1983, 243-258.

7. Local maximum property and q-plurisubharmonic functions in uniform algebras, J. Math. Anal. Appl. 115 (1986), 105-130.

8. E. Stout, The theory of uniform algebras, Bogden and Quigely, Tarrytown-on-Hudson, 1971.

9. T. Tonev, New relations between Sibony-Basener boundaries, Lecture Notes in Math., vol. 1277, Springer-Verlag, New York, 1987, 256-262.

10. __ General complex-analytic structures in uniform algebra spectra, preprint, Sofia, 1987.

11. _ Multi-dimensional analytic structures and uniform algebras, Houston J. Math. 17 (1991).

12. J. Wermer, Banach algebras and several complex variables, Graduate Texts in Math., Springer-Verlag, Berlin and New York, 1976.

Mathematics Department, The University of Toledo, Toledo, Ohio 43606-3390 